Pacific Journal of Mathematics

QUASI-UNIFORMITIES WITH A TRANSITIVE BASE 


\title{
QUASI-UNIFORMITIES WITH A TRANSITIVE BASE
}

\author{
Peter Fletcher and William F. Lindgren
}

\begin{abstract}
Every topological space admits at least one compatible transitive quasi-uniformity, and among the compatible transitive quasi-uniformities there is always a finest one. It is shown that a topological space is compact if and only if its finest transitive quasi-uniformity is precompact. A $T_{1}$ space $X$ whose fine quasi-uniformity is transitive is quasi-metrizable if and only if $X$ has a $\sigma-Q$-base. The upper semi-continuous quasi-uniformity of any topological space is transitive and countably precompact and a space $X$ is almost realcompact if and only if it has a compatible almost complete countably precompact transitive quasi-uniformity. Consequently a space is almost realcompact if and only if its upper semi-continuous quasi-uniformity is almost complete. The latter result is the natural analogue of the result of Shirota that a space $X$ is realcompact if and only if it is complete in the structure $C(X)$.
\end{abstract}

The concept of a quasi-uniformity on a set $X$ was introduced by L. Nachbin. If $X$ is a nonempty set and $\mathscr{U}$ is a filter on $X \times X$, then $\mathscr{Q}$ is a quasi-uniformity on $X$ if and only if

(i) for each $U \in \mathscr{U}, \Delta=\{(x, x): x \in X\} \subset U$

(ii) for each $U \in \mathscr{U}$, there is a $V \in \mathscr{Q}$ such that $V \circ V \subset U$. A (sub)base $\mathscr{B}$ for $\mathscr{C}$ is transitive provided that for each $B \in \mathscr{B}$, $B \circ B=B$. A quasi-uniformity with a transitive base is called a transitive quasi-uniformity.

It is not true that every quasi-uniformity is transitive; indeed if a uniformity $\mathscr{C}$ has a transitive base, then the resulting topology $\mathscr{T}_{x}$ is 0 -dimensional. It might appear reasonable to conjecture that every quasi-uniformity has either a symmetric or transitive base, but we give a simple counterexample to this conjecture as well. Nevertheless, there is some justification for the point of view that the insistence upon symmetry inherent in the axioms for a uniformity has obscured the fact that transitivity also plays an important role in general topology.

In section two we review the known methods of constructing a compatible quasi-uniformity for an arbitrary topological space; and we show that every known method may be considered as a special case of a very general method for constructing compatible quasi-uniformities which, in fact, may be used to construct every compatible transitive quasi-uniformity.

In the third section of the paper, we consider topological spaces whose fine quasi-uniformity is transitive. 
The fourth section of this paper concerns precompact and totally bounded transitive quasi-uniformities. For uniform spaces the concepts of total boundedness and precompactness are equivalent; however for quasi-uniform spaces precompactness is implied by total boundedness and is in general a weaker condition. A useful property of the Pervin quasi-uniformity $\mathscr{P}$ is that for any topological space this quasi-uniformity is totally bounded. We construct a transitive quasi-uniformity which contains the Pervin quasi-uniformity and which is always precompact. It follows that the Pervin quasi-proximity class of any infinite Hausdorff space contains two precompact members. Some of $\S 4$ is devoted to translations of known results into the setting of quasiuniform spaces. A typical result of this kind is that the locally finite covering quasi-unformity $\mathscr{L} \mathscr{F}$ of a Tychonoff space is precompact if and only if $\mathscr{L} \mathscr{F}=\mathscr{P}$. One of the most startling translations is the result, mentioned above, that a topological space is campact if and only if every compatible transitive quasi-unifomity is precompact. This result suggests the possibility that every topological space has a compatible complete transitive quasi-uniformity. At present the best theorem along these lines appears to be that every orthocompact topological space has a compatible complete transitive quasi-uniformity.

The last section concerns complete and almost complete transitive quasi-uniformities. It follows from our results and those of [10] that a normal countably paracompact space is realcompact if and only if its upper semi-continuous quasi-uniformity is complete. Consequently for a discrete space of measurable cardinality the upper semi-continuous quasi-uniformity is not complete, whereas the finest compatible transitive quasi-uniformity is the discrete structure which is complete.

Throughout this paper, if $\mathscr{C}$ is an open cover of a topological space $(X, \mathscr{T})$ and $x \in X$, then $A_{x}^{\mathscr{C}}$ denotes $\bigcap\{C \in \mathscr{C}: x \in C\}$.

2. Construction of quasi-uniformities. At present, there are two general methods of constructing a compatible quasi-uniformity for an arbitrary topoligical space. Although we shall show that one of these methods can in fact be considered as a special case of the other, the two methods are conceptually different in that one method depends upon the construction of a transitive base while the other does not. Moreover only one method has a natural analogue in the construction of a uniformity for a completely regular space. We begin by recalling these methods of construction.

(i) Covering quasi-uniformities. A $Q$-cover of a topological space $(X, \mathscr{T})$ is an open cover $\mathscr{C}$ of $X$ such that if $x \in X$, then $A_{x}^{\mathscr{E}} \in \mathscr{T}$ [25]. Let $\mathscr{A}$ be a collection of $Q$-covers of a topological space $(X, \mathscr{T})$ such that if $x \in A \in \mathscr{T}$, then there is $\mathscr{C} \in \mathscr{A}$ such that $A_{x}^{\mathscr{C}} \subset A$. For 
each $\mathscr{C} \in \mathscr{A}$, let $U_{\mathscr{C}}=\bigcup\left\{\{x\} \times A_{x}^{\mathscr{C}}: x \in X\right\}$ and let $\mathscr{S}=\left\{U_{\mathscr{C}}: \mathscr{C} \in \mathscr{A}\right\}$. Then $\mathscr{S}$ is a transitive subbase for a compatible quasi-uniformity $\mathscr{C}_{\propto}$ for $(X, \mathscr{T})$ [6, Theorem 1]. The quasi-uniformity $\mathscr{C}_{\propto}$ is called the covering quasi-uniformity for $(X, \mathscr{T})$ with respect to $\mathscr{A}$. If $\mathscr{A}$ is, respectively, the collection of all ( $Q$-covers, point finite open covers, locally finite open covers, finite open covers,) then $\mathscr{C}_{\alpha}$ is the fine transitive, point finite covering, locally finite covering, Pervin quasiuniformity for $(X, \mathscr{T})$ and is denoted by $\mathscr{F} \mathscr{T}, \mathscr{P} \mathscr{F}, \mathscr{L} \mathscr{F}, \mathscr{P}$ respectively. Since every transitive quasi-uniformity is a covering quasiuniformity [6, Theorem 2], the fine transitive quasi-uniformity is, as the name suggests, the finest compatible transitive quasi-uniformity for a given topological space $(X, \mathscr{T})$. It is shown in [26] that the Pervin quasi-uniformity is the same as the quasi-uniformity first defined by A. Császár in [4]. Moreover the quasi-uniformity defined by R. Nielsen and C. Sloyer in [22] is also the Pervin quasi-uniformity.

(ii) Upper semi-continuous quasi-uniformities. Let $(X, \mathscr{T})$ be a topological space and let $F$ be a collection of upper semi-continuous functions which cotains the collection of all characteristic functions on closed sets. For each $f \in F$ and each $\varepsilon>0$, let $U_{(f, \varepsilon)}=\{(x, y) \in$ $X \times X: f(y)-f(x)<\varepsilon\}$. Then $\mathscr{B}=\left\{U_{(f, s)}: f \in F, \varepsilon>0\right\}$ is a subbase for a compatible quasi-uniformity $\mathscr{C}_{F}$ for $(X, \mathscr{T})$ [7, Theorem 1]. If $U$ is the collection of all upper semi-continuous functions then $\mathscr{C}_{U}$ is called the upper semi-continuous quasi-uniformity and is denoted by $26 \mathscr{S C C}$. It is clear that in general the subbase $\mathscr{B}$ for $\mathscr{H} \mathscr{S} \mathscr{C}$ need not be a transitive subbase.

Definition [18]. An open spectrum $\boldsymbol{a}$ in a set $X$ is a sequence $\left\{A_{n}\right\}$ of open subsets of $X$ indexed by the integers, $Z$, such that for each $n \in Z, A_{n} \subset A_{n+1}, \bigcap_{n \in Z} A_{n}=\varnothing$ and $\mathrm{U}_{n \in Z} A_{n}=X$.

Each open spectrum $\boldsymbol{a}$ is a $Q$-cover. There is an integer $n$ such that $A_{n}=X$ if and only if $\boldsymbol{a}$ is a point-finite open cover. In this case we call $\boldsymbol{a}$ a point-finite open spectrum. If $\boldsymbol{a}$ is an open spectrum, $x \in X$ and $n$ is that integer for which $x \in A_{n}-A_{n-1}$, then $A_{x}^{a}=A_{n}$ and $U_{a}=\bigcup_{x \in X}\{x\} \times A_{x}^{a}={ }_{n \in} \bigcup_{Z}\left(A_{n}-A_{n-1},\right) \times A_{n}$.

Theorem 2.1. Let $\mathscr{A}$ be the collection of all open spectra in a space $X$. Then $\mathscr{W}=\mathscr{W} \mathscr{S} \mathscr{C}$.

Proof. Let $U$ be the collection of all upper semi-continuous functions. It suffices to show that $\left\{U_{a}: \boldsymbol{a} \in \mathscr{A}\right\}$ and $\left\{U_{(f, s)}: \varepsilon>0, f \in U\right\}$ are equivalent subbases. Let $f \in U$ and $\varepsilon>0$ be given; take $x_{0} \in X$. Set $A_{n}=\left\{x: f(x)<f\left(x_{0}\right)+(n+1) \varepsilon\right\}$ and set $\boldsymbol{a}=\left\{A_{n}\right\}$. Let $(x, y) \in U_{a}$. There is an integer $n$ such that $f\left(x_{0}\right)+(n-1) \varepsilon \leqq f(x) \leqq f\left(x_{0}\right)+n \varepsilon$. Then $y \in U_{a}(x)=A_{x}^{a}=A_{n}$ so that $f(y)<f\left(x_{0}\right)+(n+1) \varepsilon$. Hence $f(y)-$ 
$f(x)<f\left(x_{0}\right)+(n+1) \varepsilon-\left(f\left(x_{0}\right)+n \varepsilon\right)=\varepsilon$ and $(x, y) \in U_{(f, \varepsilon)}$.

Let $a \in \mathscr{A}$. For each $x \in X$, let $n$ be that integer for which $x \in$ $A_{n}-A_{n-1}$ and define $f(x)=n \varepsilon$. Let $(x, y) \in U_{(f, \varepsilon)}$; then $f(y)<f(x)+\varepsilon$. Since $x \in A_{n}-A_{n-1}, f(y)<f(x)+\varepsilon=(n+1) \varepsilon$. Hence $y \in A_{n}$ and $(x, y) \in$ $\left(A_{n}-A_{n-1}\right) \times A_{n} \subset U_{a}$.

Corollary. Let $X$ be a topological space. Then wSPC $\subset \mathscr{F}$. If $X$ is countably compact then each open spectrum is a point-finite open spectrum and $\mathscr{U} \mathscr{S} \mathscr{C} \subset \mathscr{P} \mathscr{F}$.

We omit the proof of the following theorem which is similar to the proof of Theorem 2.1.

THEOREM 2.2. Let $(X, \mathscr{T})$ be a topological space, let $U$ be the collection of all upper semi-continuous functions which are bounded above and let $\mathscr{A}$ be the collection of all point finite open spectra. Then $\mathscr{U}_{U}=\mathscr{U}_{\mathscr{S}}$.

If in the above theorem $U$ is taken to be the collection of all bounded upper semi-continuous functions and $\mathscr{A}$ is taken to be the collection of all finite open covers, then $\mathscr{C}_{U}=\mathscr{C}_{W}[12$, Theorem 2].

LEMmA. Let $\mathscr{Q}$ be a quasi-uniformity on a set $X$ and suppose that $\mathscr{Q}$ is a transitive quasi-uniformity with a countable base $\alpha=$ $\left\{U_{n}\right\}_{n=1}^{\infty}$ such that for each positive integer $n, U_{n+1} \circ U_{n+1}=U_{n}$. Then $\mathscr{Q}$ is the fine quasi-uniformity for $\mathscr{T}_{\mathscr{W}}$ and $\alpha$ consists of a single transitive entourage.

Proof. Let $\beta$ be a transitive base for $\mathscr{Z}$ and let $n$ be a positive integer. Since $\alpha$ and $\beta$ are bases for $\not 6$, there exists a positive integer $m$ with $m \geqq n$ and $U \in \beta$ such that $U_{m} \subset U \subset U_{n}$. Since $U$ is a transitive relation on $X, U_{m} \circ U_{m}=U_{m-1} \subset U \subset U_{n}$. It follows by induction that $U_{n}=U$. Thus each member of $\alpha$ is a transitive relation on $X$ so that $\alpha=\left\{U_{1}\right\}=\{U\}$. It follows that $\mathscr{C}$ is the fine quasi-uniformity for $\mathscr{T}_{z}[8$, Theorem 3.3].

EXAMPLE 2.3. A quasi-uniformity which has neither a symmetric nor a transitive base. Let $\boldsymbol{R}$ be the set of real numbers, for each positive integer $n$, let $U_{n}=\left\{(x, y): y-x<1 / 2^{n-1}\right\}$ and let $\mathscr{Q}$ be the quasi-uniformity on $\boldsymbol{R}$ generated by the base $\left\{U_{n}\right\}_{n=1}^{\infty}$. By the above lemma, it is evident that $\mathscr{C}$ is not a transitive quasi-uniformity. It is also evident that $\mathscr{U}$ is not a uniformity.

Question. Does there exist a method of constructing a compatible 
quasi-uniformity for a topological space such that

(i) the method of construction obtains for any topological space

(ii) there is a topological space for which the quasi-uniformity of the construction does not have a transitive base?

In particular is the fine (universal) quasi-uniformity of a topological space necessarily a transitive quasi-uniformity?

\section{Transitive spaces.}

DEFINITION. A topological space $(X, \mathscr{T})$ is a transitive space provided that the fine quasi-uniformity for $(X, \mathscr{T})$ is the fine transitive quasi-uniformity $\mathscr{F} \mathscr{T}$.

Proposition 3.1. Let $X=\left\{x_{i}\right\}_{i=1}^{\infty}$ be a countable set and let $\mathscr{T}$ be a $T_{1}$ topology for $X$. Then $(X, \mathscr{T})$ is a metacompact transitive space.

Proof. Let $\mathscr{U}$ be the fine quasi-uniformity and let $U \in \mathscr{U}$. For each positive integer $i$, let $V_{i+1}=\operatorname{int} U\left(x_{i+1}\right)-\left\{x_{1}, x_{2}, \cdots, x_{i}\right\}$ and let $V_{1}=\operatorname{int} U\left(x_{1}\right)$. Then $\mathscr{R}=\left\{V_{i}\right\}_{i=1}^{\infty}$ is a point-finite open cover of $X$ and for each $x_{i} \in X, U_{\mathscr{R}}\left(x_{i}\right)=A_{x_{i}}^{\mathscr{R}} \subset V_{i} \subset U\left(x_{i}\right)$. Thus $U_{\mathscr{R}} \subset U$ and hence $\mathscr{P} \mathscr{F}=\mathscr{U}$. It is evident that $(X, \mathscr{T})$ is metacompact.

Topological spaces with a finite topology are uniquely quasi-uniformizable and hence are transitive spaces [2, Theorem 3.4]. Conversely if $(X, \mathscr{T})$ is $R_{1}$ (in particular if $(X, \mathscr{T})$ is either Hausdorff or regular) and $(X, \mathscr{T})$ has only one compatible quasi-uniformity, then $\mathscr{T}$ is finite [19, Theorem 2.3]. Another example of a transitive space may be found in [9, Example 2.2].

Proposition 3.2. Let $(X, \mathscr{T})$ be a transitive space. Then $(X, \mathscr{T})$ has only one compatible quasi-uniformity if and only if every $Q$-cover of $X$ is finite.

Proof. Since $\mathscr{P}$ is totally bounded, by Lemma 4.1, if $\mathscr{P}=\mathscr{F} \mathscr{T}$, then every $Q$-cover of $X$ is finite. Again by Lemma 4.1, if every $Q$ cover of $X$ is finite, then $\mathscr{F} \mathscr{T}$ is totally bounded. Since $\mathscr{F} \mathscr{T}$ is assumed to be the fine quasi-uniformity the result now follows from [20, Corollary 3.6].

It is shown in [20] that if $(X, \mathscr{T})$ is a topological space with the property that every $Q$-cover of $X$ is finite, then $(X, \mathscr{T})$ is hereditarily compact. Note, however, that the real line with the cofinite topology is an example of a space with infinitely many open sets which satisfies the conditions of Proposition 3.2. 
DEFINITION [25]. A topological space $(X, \mathscr{T})$ is orthocompact ("has property $Q$ " in terms of [25]) provided that every open cover of $X$ has an open $Q$-refinement.

Proposition 3.3. Let $(X, \mathscr{T})$ be an orthocompact space. Then $\mathscr{F} \mathscr{T}$ contains every compatible uniformity for $(X, \mathscr{T})$.

Proof. Let $\mathscr{Q}$ be a compatible uniformity for $(X, \mathscr{T})$ and let $U \in \mathscr{K}$. Let $W$ be an open symmetric entourage such that $W \circ W \subset U$, let $\mathscr{C}=\{W(x): x \in X\}$ and let $\mathscr{R}$ be a $Q$-refinement of $\mathscr{C}$. Then, for each $x \in X, U_{\mathscr{E}}(x) \subset$ st $(x, \mathscr{C}) \subset U(x)$. Thus $U_{\mathscr{R}} \subset U$ so that $\mathscr{U} \subset \mathscr{F} \mathscr{T}$.

Definition. Let $(X, \mathscr{T})$ be a topological space and let $\mathscr{C}$ be a collection of open sets such that if $x \in X$, then $\bigcap\{C \in \mathscr{C}: x \in C\} \in \mathscr{T}$. Then $\mathscr{C}$ is a $Q$-collection. If $\beta$ is a base for $\mathscr{T}$ and there is a sequence $\left\{\beta_{i}\right\}_{i=1}^{\infty}$ of $Q$-collections such that $\beta=\bigcup_{i=1}^{\infty} \beta_{i}$, then $\beta$ is a $\sigma-Q$-base for $\mathscr{T}$.

It is shown in [9] that if $(X, \mathscr{T})$ is a topological space and $\mathscr{T}$ has a $\sigma-Q$-base, then $(X, \mathscr{T})$ is quasi-metrizable. For transitive spaces a complete analogue of the Nagata-Smirnov metrization theorem obtains.

THeOREM 3.4. Let $(X, \mathscr{T})$ be a $T_{1}$ transitive space. Then $(X, \mathscr{T})$ is quasi-metrizable if and only if $\mathscr{T}$ has a $\sigma-Q$-base.

Proof. Suppose that $(X, \mathscr{T})$ is quasi-metrizable. Then $(X, \mathscr{T})$ has a compatible quasi-uniformity $\mathscr{Q}$ with a countable base $\beta=$ $\left\{\beta_{i}\right\}_{i=1}^{\infty}[16$, page 185]. Since $\mathscr{B} \subset \mathscr{Q} \subset \mathscr{F} \mathscr{T}$, there are transitive entourages $B_{1}^{T}, B_{2}^{T}, \cdots \in \mathscr{F} \mathscr{T}$ such that for each positive integer $i$, $B_{i}^{T} \subset B_{i}$. Let $\beta^{T}=\left\{B_{i}^{T}\right\}_{i=1}^{\infty}$ and let $\mathscr{Q}^{T}$ be the quasi-uniformity for which $\beta^{T}$ is a subbase. Clearly $\mathscr{\mathscr { C }} \subset \mathscr{Q}^{T} \subset \mathscr{F} \mathscr{T}$. Consequently $\mathscr{T}=$ $\mathscr{T}_{\mathscr{H}} \subset \mathscr{I}_{\mathscr{Z}^{T}} \subset \mathscr{T}_{\mathscr{T} \mathscr{T}}=\mathscr{T}$. Thus $\beta^{T}$ is a countable transitive subbase for a quasi-metrizable quasi-uniformity for $(X, \mathscr{T})$. Let $\left\{U_{i}\right\}_{i=1}^{\infty}$ be the countable base generated by $\beta^{T}$. For each positive integer $i$, let $\beta_{i}=$ $\left\{U_{i}(x): x \in X\right\}$. Then $\beta_{i}$ is an open cover of $X$. Let $x \in X$ and let $y \in A_{x}^{\beta_{i}}$. Let $z \in U_{i}(y)$ and let $t \in X$. Suppose that $x \in U_{i}(t)$. Then $y \in A_{x}^{\beta_{i}} \subset U_{i}(t)$ so that $z \in U_{i}(y) \subset U_{i}^{\circ} U_{i}(t)$. Consequently $z \in A_{x}^{\beta_{i}}$ so that $U_{i}(y) \subset A_{x}^{\beta_{i}}$. It follows that $A_{x}^{\beta_{i}} \in \mathscr{T}$. For each positive integer $i, \beta_{i}$ is a $Q$-collection and hence $\mathscr{T}$ has a $\sigma-Q$-base.

4. Precompact and totally bounded transitive quasi-uniformities.

Definition. Let $(X, \mathscr{U})$ be a quasi-uniform space. Then $\mathscr{C}$ is 
precompact (countably precompact) provided that if $U \in \mathscr{Q}$, then there is a finite (countable) subset $F$ of $X$ such that $U(F)=X$.

Definition. Let $(X, \mathscr{C})$ be a quasi-uniform space. Then $\mathscr{C}$ is totally bounded provided that if $U \in \mathscr{Q}$, then there is finite collection $\left\{A_{i}\right\}_{i=1}^{n}$ of subsets of $X$ such that $\bigcup\left\{A_{i}: 1 \leqq i \leqq n\right\}=X$ and such that for $1 \leqq i \leqq n, A_{i} \times A_{i} \subset U$.

Lemma 4.1. Let $(X, \mathscr{T})$ be a topological space and let $\mathscr{U}_{\mathscr{A}}$ be a compatible covering quasi-uniformity. A necessary and sufficient condition that $\mathscr{U}_{\mathscr{A}}$ be totally bounded is that each member of $\mathscr{A}$ be a finite open cover.

Proof. Necessity follows with minor modifications from the proof of [20, Theorem 3.3]. Now suppose that each member of $\mathscr{A}$ is a finite open cover and let $\mathscr{B}$ be the collection of all finite open covers.

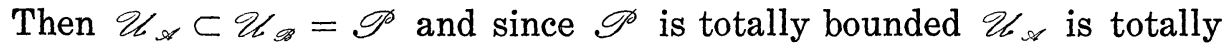
bounded as well.

Note that if $\mathscr{U}_{\mathscr{A}}$ is a precompact covering quasi-uniformity, then every member of $\mathscr{A}$ has a finite subcover. Certainly it is possible for a collection of $Q$-covers, $\mathscr{A}$, to have the property that every member of $\mathscr{A}$ has a finite subcover without $\mathscr{C} \propto$ 's being precompact. For example the collection of all $Q$-covers of a space $X$ which contain $\{X\}$ as a member generates a covering quasi-uniformity $\mathscr{U}_{\mathscr{A}}$ which is not in general precompact.

Definition. A collection $\mathscr{A}$ of $Q$-covers of a space $X$ is an esplanade for $X$ provided that

(i) if $\left\{\mathscr{C}_{i}\right\}_{i=1}^{n}$ is a finite subset of $\mathscr{A}$, then $\bigcup_{i=1}^{n} \mathscr{C}_{i} \in \mathscr{A}$.

(ii) if $\mathscr{C} \in \mathscr{A}$, then $\left\{A_{x}^{\mathscr{E}}: x \in X\right\} \in \mathscr{A}$.

(iii) if $x \in U \in \mathscr{T}$, there exists $\mathscr{C} \in \mathscr{A}$ such that $A_{x}^{\mathscr{C}} \subset U$.

We note that if $\mathscr{A}$ is the collection of point finite (locally finite, $Q)$ open covers of $X$, then $\mathscr{A}$ is an esplanade for $X$, and that in general $\mathscr{A}$ is an esplanade if and only if $\left\{U_{\mathscr{E}}: \mathscr{C} \in \mathscr{A}\right\}$ is a base for a compatible quasi-uniformity.

Proposition 4.2. Let $X$ be a topological space and let $\mathscr{A}$ be an esplanade for $X$. Then $\mathscr{C}_{\infty}$ is (countably) precompact if and only if every member of $\mathscr{A}$ has a finite (countable) subcover.

Proof. Suppose that every member of $\mathscr{A}$ has a finite (countable) subcover. Let $U$ be a basic entourage of $\mathscr{C}_{\mathscr{A}}$. There is a finite subcollection $\left\{\mathscr{C}_{i}\right\}_{i=1}^{n}$ of $\mathscr{A}$ such that $U=\bigcap_{i=1}^{n} U_{\mathscr{C}_{i}}$. Let $\mathscr{C}=\bigcup_{i=1}^{n} \mathscr{C}_{i}$. 
Then $U=\bigcap_{i=1}^{n} U_{\mathscr{E}_{i}}=U_{\mathscr{E}}$ and $\mathscr{C} \in \mathscr{A}$. Thus $\{U(x): x \in X\}=\left\{A_{x}^{\mathscr{C}}: x \in X\right\} \in$ $\mathscr{A}$. By hypothesis $\{U(x): x \in X\}$ has a finite (countable) subcover. Thus $\mathscr{C}_{\mathscr{A}}$ is (countably) precompact.

One of the most useful properties of the Pervin quasi-uniformity $\mathscr{P}$ is that $\mathscr{P}$ is always totally bounded. Nevertheless total boundedness is sometimes of overwhelming strength so that is desirable to have a method of constructing a compatible quasi-uniformity for an arbitrary topological space which is always precompact but which is not necessarily totally bounded. We will show that if $(X, \mathscr{T})$ is a topological space and $\mathscr{A}$ is the collection of all point-finite open spectra, then $\mathscr{U}_{\mathscr{A}}$ is precompact. Note that since $\mathscr{A}$ is not an esplanade Proposition 4.2 is not applicable.

In the following Lemma, we will consider only point-finite open spectra. Let $N$ denote the set of all positive integers and let the point-finite open spectrum $\boldsymbol{a}_{n}$ be given by $\boldsymbol{a}_{n}=\{A(n, j): j \in N\}$. Set $\mathscr{A}_{n}=\bigcup_{i=1}^{n} \boldsymbol{a}_{i}$. For convenience, for each $n$, suppose $A(n, 1)=X$ and for each $n, j, A(n, j) \supset A(n, j+1)$. (I.e. relabel these spectra by the positive integers.)

Lemma. Let $X$ be a topological space. For each positive integer $n$, for each finite collection $\left\{\boldsymbol{a}_{1}, \boldsymbol{a}_{2}, \cdots, \boldsymbol{a}_{n}\right\}$ of point-finite open spectra and for each $Y \subset X$, the open collection $\left\{A_{y}^{\aleph_{n}}: y \in Y\right\}$ contains a finite subcover of $Y$.

Proof. The proof is by induction on $n$. Case $n=1$. Let a subspace $Y$ and a point-finite open spectrum $a_{1}$ in $X$ be given. Set $M=$ $\inf \{j$ : for some $y \in Y, y \in A(1, j)-A(1, j+1)\}$. Then if $y \in A(1, M)-$ $A(1, M+1), A_{y}^{\mathscr{N}_{1}}=A(1, M) \supset Y$.

Let a subspace $Y$ and a collection $\left\{\boldsymbol{a}_{1}, \boldsymbol{a}_{2}, \cdots, \boldsymbol{a}_{n}, \boldsymbol{a}_{n+1}\right\}$ of pointfinite open spectra in $X$ be given. Then by the inductive hypothesis, there exist $y_{i} \in Y(1 \leqq i \leqq m)$ such that $\bigcup_{i=1}^{m} A_{y_{i}}^{*} \supset Y$. For each $i$, there exists a $j(i)$ such that $y_{i} \in A(n+1, j(i))-A(n+1, j(i)+1)$. Set $M=$ greatest $j(i)$. Then we have

$$
\begin{aligned}
\bigcup_{i=1}^{m} A_{y_{i}}^{\mathscr{s}} n+1= & \bigcup_{i=1}^{m}\left(A_{y_{i}}^{\mathscr{s}} n \cap A(n+1, j(i))\right) \supset \bigcup_{i=1}^{m}\left(A_{y_{i}}^{\mathscr{s}} n \cap A(n+1, M)\right) \\
& \supset Y \cap A(n+1, M) .
\end{aligned}
$$

Once again by the inductive hypothesis, for each $k<M$, there exist $y(k, i),\left(i=1,2, \cdots, M_{k}\right)$ with

$$
y(k, i) \in(A(n+1, k)-A(n+1, k+1)) \cap Y
$$

such that

$$
\bigcup_{t=1}^{m_{k}} A_{y}^{\mho^{*}}(k, t) \supset(A(n+1, k)-A(n+1, k+1)) \cap Y .
$$


Hence for each $k$,

$$
\begin{aligned}
\bigcup_{t=1}^{m_{k}} A_{y}^{\varkappa_{n+1}}(k, t)= & \bigcup_{t=1}^{m_{k}}\left(A_{y}^{\varkappa_{n+1}}(k, t) \cap A(n+1, k)\right) \\
& \supset Y \cap(A(n+1, k)-A(n+1, k+1)) .
\end{aligned}
$$

Since $Y=Y \cap A(n+1, M) \cup \bigcup_{k<m}(Y \cap(A(n+1, k)-A(n+1, k+1)))$,

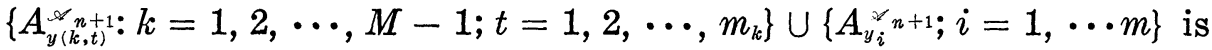
the required finite subcover of $\left\{A_{y}^{\mathscr{A}_{n+1}}: y \in Y\right\}$.

THEOREM 4.3. Let $(X, \mathscr{T})$ be a topological space and let $\mathscr{A}$ be the collection of all point- finite open spectra for $(X, \mathscr{T})$. Then $\mathscr{U} \&$ is precompact.

Proof. Let $U$ be a basic entourage of $\mathscr{Q}_{\mathscr{A}}$. There exists $\left\{\boldsymbol{a}_{i}\right\}_{i=1}^{n} \subset$ $\mathscr{A}$ such that $U=\bigcap_{i=1}^{n} U_{a_{i}}$. By the preceding lemma there is a finite set $F \subset X$ such that $U(F)=\left[\bigcap_{i=1}^{n} U_{a_{i}}\right](F)=X$.

Proposition 4.4. Let $(X, \mathscr{T})$ be an orthocompact space. Then the following statements are equivalent.

(i) Every compatible quasi-uniformity is countably precompact

(ii) $(X, \mathscr{T})$ is Lindelöf.

It is known that a $T_{1}$ topological space $(X, \mathscr{T})$ admits a compatible totally bounded non-archimedean quasi-metric if and only if $(X, \mathscr{T})$ satisfies the second axiom of countability [9, Theorem 3.3]. Nevertheless the authors have been unable to understand why an orthocompact $T_{1}$ space which admits a compatible totally bounded non-archimedean quasi-metric necessarily satisfies (i) of Proposition 4.4.

By Proposition 4.2, the point-finite covering quasi-uniformity $\mathscr{P} \mathscr{F}$ of a topological space $(X, \mathscr{T})$ is precompact if and only if every pointfinite open cover has a finite subcover. If $(X, \mathscr{T})$ is countably compact, then $\mathscr{L} \mathscr{F}$ is totally bounded and if $(X, \mathscr{T})$ is a regular space then $\mathscr{P} \mathscr{F}$ is pecompact if and only if $(X, \mathscr{T})$ is countably compact [14, Theorem 2]. It is shown in [2] that a topological space is countably compact if and only if $\mathscr{E} \mathscr{S} \mathscr{C}$ is precompact. A considerably simpler proof of this result follows from Theorem 2.1.

Proposition 4.5. Let $(X, \mathscr{T})$ be a topological space. Then the following statements are equivalent.

(i) $(X, \mathscr{T})$ is countably compact.

(ii) Every countable Q-cover has a finite subcover.

(iii) $\mathscr{U} \mathscr{S} \mathscr{C}$ is precompact.

(iv) Every upper semi-continuous function is bounded above. 
Proof. Throughout the proof let $\mathscr{A}$ be the collection of all open spectra and let $\mathscr{B}$ be the collection of all point-finite open spectra. It is evident that (i) $\Rightarrow$ (ii). (ii) $\Rightarrow$ (iii): If every countable $Q$-cover has a finite subcover, then every open spectrum is a point-finite open spectrum. In this case $\mathscr{U}_{\mathscr{S}} \mathscr{C}=\mathscr{U}_{\infty}=\mathscr{U}_{\mathscr{H}}$ which is precompact by Theorem 4.3. (iii) $\Rightarrow$ (iv): By Theorem 2.1, $\mathscr{U} \mathscr{S} \mathscr{C}=\mathscr{U} \mathscr{A}$ Evidently, if $\mathscr{C}_{\mathscr{A}}$ is precompact, then each $\boldsymbol{a} \in \mathscr{A}$ is a point-finite open spectrum. Let $f$ be an upper semi-countinuous function. Then $\left\{f^{-1}(-\infty, n): n \in Z\right\}$ is an open spectrum. Consequently $f$ is bounded above. (iv) $\Rightarrow$ (i). [15, Theorem 1].

Proposition 4.6. Let $(X, \mathscr{T})$ be a Tychonoff space. Then the following statements are equivalent.

(i) $\mathscr{L} \mathscr{F}$ is precompact.

(ii) $\mathscr{L} \mathscr{F}$ is totally bounded.

(iii) $\mathscr{L} \mathscr{F}=\mathscr{P}$.

(iv) $(X, \mathscr{T})$ is pseudocompact.

(v) Every locally finite open cover has finite subcover.

(vi) Every locally finite open cover is finite.

Proof. (i) $\Leftrightarrow$ (v): Proposition 4.2. (iv) $\Leftrightarrow$ (v) $\Leftrightarrow$ (vi): [17, Theorem 1]. (vi) $\Leftrightarrow$ (ii): Lemma 4.1. (ii) $\Leftrightarrow$ (iii): $\mathscr{L} \mathscr{F}$ is a member of the Pervin quasi-proximity class since $\mathscr{P} \subset \mathscr{L} \mathscr{F}$ and $\mathscr{P}$ is the unique totally bounded member of its quasi-proximity class [12, Theorem 1].

In a normal Hausdorff space pseudocompactness is equivalent to countable compactness. Thus in a normal Hausdorff space, the conditions of the previous propositions are equivalent.

The full strength of total boundedness is not apparent in the previous propositions. We have included the next result in order to point out that total boundedness can sometimes be a much stronger condition than precompactness.

Proposition 4.7. Let $(X, \mathscr{T})$ be a Hausdorff space. Then the following statements are equivalent.

(i) $\mathscr{C} \mathscr{S} \mathscr{C}$ is totally bounded.

(ii) Every upper semi-continuous function is bounded.

(iii) Every point finite open cover is finite.

(iv) $\mathscr{P} \mathscr{F}$ is totally bounded.

(v) $X$ is a finite set.

Proof. (i) $\Leftrightarrow$ (ii): [12, Theorem 2] and [13, Corollary 3]. (ii) $\Rightarrow$ (iii): [15, Theorem 2]. (iii) $\Leftrightarrow$ (iv): Lemma 4.1. (iii) $\Rightarrow(v)$ : Each infinite Hausdorff space has countably infinite pairwise disjoint collection $\mathscr{C}$ of open sets. Then $\mathscr{C} \cup\{X\}$ is an infinite point finite open cover. 
The equivalence of Proposition 4.5 (i) and 4.5 (ii) suggests the possibility that a topological space is compact (Lindelöf) if and only if every $Q$-cover of $X$ has a finite (countable) subcover. For the Lindelof property, the matter is to the authors' knowledge unresolved. For compactness, a better result is already in the literature.

THEOREM 4.8. [1, Theorem 7.1]. Let $(X, \mathscr{T})$ be a topological space. Then $(X, \mathscr{T})$ is compact if and only if every open cover which is well-ordered by set inclusion has a finite subcover.

Corollary. Let $(X, \mathscr{T})$ be a topological space. Then the following statements are equivalent:

(i) $(X, \mathscr{T})$ is compact.

(ii) Every compatible quasi-uniformity is precompact.

(iii) Every compatible transitive quasi-uniformity is precompact.

Corollary. Let $(X, \mathscr{T})$ be a countably compact space which is not compact. Then $\mathscr{U} \mathscr{S} \mathscr{C} \varsubsetneqq \mathscr{F} \mathscr{T}$. If $(X, \mathscr{T})$ is regular, then $\mathscr{P} \mathscr{F} \varsubsetneqq \mathscr{F} \mathscr{T}$.

5. Complete and almost complete quasi-uniformities. Let $(X, \mathscr{C})$ be a quasi-uniform space and let $\mathscr{F}$ be a filter on $X$. Then $\mathscr{F}$ is Cauchy provided that if $V \in \mathscr{U}$, there is $p \in X$ such that $V(p) \in$ $\mathscr{F}$. The space $(X, \mathscr{K})$ is complete provided that every Cauchy filter converges and the space $(X, \mathscr{C})$ is almost complete provided that every open Cauchy filter has a cluster point. A collection of subsets $\mathscr{C}$ has a cluster point provided that $\bigcap\{\bar{A}: A \in \mathscr{C}\} \neq \varnothing$. A filter $\mathscr{F}$ on a topological space $(X, \mathscr{T})$ has the countable subcollection intersection property (C.C.I.P.) if every countable subcollection has a cluster point. The space is almost realcompact if every open ultrafilter with C.C.I.P. has a cluster point. We refer to [5] and [10] for general properties of almost complete and almost realcompact spaces. The following lemma is an immediate consequence of Theorem 2.1.

Lemma. For any topological space, $\mathscr{U} \mathscr{S} \mathscr{C}$ is a countably precompact transitive quasi-uniformity.

THEOREM 5.1. A topological space is almost realcompact if and only if there is a compatible almost complete countably precompact transitive quasi-uniformity.

Proof. Suppose first that $(X, \mathscr{T})$ is an almost realcompact topological space. By the previous lemma it suffices to show that $\mathscr{U} \mathscr{S} \mathscr{C}$ is almost complete. Let $\mathscr{F}$ be a $\mathscr{U} \mathscr{S} \mathscr{C}$-Cauchy open ultrafilter. The 
filter $\mathscr{F}$ has the C.C.I.P. if and only if every upper semi-continuous function into the nonnegative real numbers is bounded on some $F \in \mathscr{F}$ [11, Theorem 1]. Let $f$ be such a function and let $\varepsilon>0$. Then there is $p \in X$ such that $U_{f, \varepsilon}(p) \in \mathscr{F}$. Evidently $f$ is bounded on $U_{(f, \varepsilon)}(p)$. Thus $\mathscr{F}$ has C.C.I.P. and is a convergent filter.

Now let $\mathscr{C}$ be an almost complete countably precompact transitive quasi-uniformity and let $\mathscr{F}$ be an open ultrafilter which has the C.C.I.P. Let $V \in \mathscr{Q}$. There is $U=U \circ U \in \mathscr{U}$ such that $U \subset V$, and there is a countable subset $A$ of $X$ such that $U(A)=X$. Suppose that for $x \in A, U(x) \notin \mathscr{F}$. Since $\mathscr{F}$ is an open ultrafilter it follows that for each $x \in A, X-\overline{U(x)} \in \mathscr{F}$. Since $\mathscr{F}$ has C.C.I.P., there is

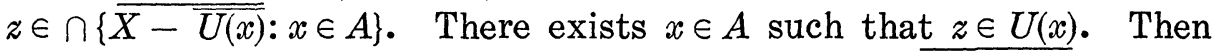
$U(z) \subset U(x)$ which contradicts the assumption that $z \in \overline{X-\overline{U(x)}}$. Thus $\mathscr{F}$ is a Cauchy filter and so $\mathscr{F}$ converges.

COROLlARY. A topological space is almost realcompact if and only if $\mathscr{U C S C}$ is almost complete.

It is known that every normal countably paracompact almost realcompact space is realcompact. If $(X, \mathscr{T})$ is a realcompact space, then the structure $C(X)$ is a complete uniformity and since $C(X) \subset \mathscr{Q} \mathscr{S} \mathscr{C}$, $\mathscr{U} \mathscr{S} \mathscr{C}$ is also complete.

CoRollary. Let $(X, \mathscr{T})$ be a normal countably paracompact space. Then $(X, \mathscr{T})$ is realcompact if and only if $\mathscr{U} \mathscr{S} \mathscr{C}$ is complete.

Let $(X, \mathscr{D})$ be a discrete space of measurable cardinality. Then the fine (quasi-)uniformity is $\mathscr{F} \mathscr{T}$ and is complete, whereas $\mathscr{U} \mathscr{S} \mathscr{C}$ is the structure $C(X)$ and is not complete.

\section{REFERENCES}

1. P. Alexandroff and P. S. Urysohn, Mémoire sur les espace topologiques compacts, Verh. Akad. Wetensch. Amsterdam, 14 (1929), 1-96.

2. C. Barnhill and P. Fletcher, Topological spaces with a unique compatible quasiuniform structure, Arch. Math., 21 (1970), 206-209.

3. J. Carlson and T. Hicks, Completeness in quasi-uniform spaces, submitted for publication.

4. A. Császár, Fondements de la topologie generale, Gauthier-Villars Paris (1960).

5. P. Fletcher and S. A. Naimpally, On almost complete and almost precompact quasiuniform spaces, Czech. Math. J., 21 (1971), 383-390.

6. P. Fletcher, On completeness of quasi-uniform spaces, Arch. Math., 22 (1971), 200204.

7. - On totally bounded quasi-uniform spaces, Arch. Math., 21 (1970), 396-401.

8. - Finite topological spaces and quasi-uniform structures, Canad. Math. Bull., 12 (1969), 771-775. 
9. P. Fletcher and W. F. Lindgren, Transitive quasi-uniformities, J. Math. Anal. Appl., 39 (1972), 397-405.

10. Z. Frolik, A generalization of realcompact spaces, Czech. Math. J., 13 (1963), 127137.

11. Z. Frolik and C. T. Liu, An embedding characterization of almost realcompact spaces, Proc. Amer. Math. Soc., 32 (1972), 294-298.

12. W. Hunsaker and W. F. Lindgren, Construction of quasi-uniformities, Math. Ann., 188 (1970), 39-42.

13. - Quasi-proximity classes of quasi-uniformities, submitted for publication. 14. K. Iséki and S. Kasahara, On pseudo-compact and countably compact spaces, Proc. Japan Acad., 33 (1957), 100-102.

15. S. Kasahara, Boundedness of semicontinuous finite real functions, Proc. Japan Acad., 33 (1957), 183-186.

16. J. L. Kelley, General topology, New York (1955).

17. J. Kerstan, Zur Characterisierung der pseudokompakten Räume, Math. Nachr., 16 (1957), 289-293.

18. S. Leader, Spectral structures and uniform continuity, Fund. Math., 60 (1967), 105-115.

19. W. F. Lindgren, Topological spaces with a unique quasi-uniform structure, Arch. Math., 22 (1971), 417-419.

20. Topological spaces with a unique compatible quasi-uniformity, Canad. Math. Bull., 14 (1971), 369-372.

21. L. Nachbin, Topology and order, D. Van Nostrand Company, Princeton, N. J. (1965).

22. R. Nielsen and C. Sloyer, Quasi-uniformizability, Math. Ann., 182 (1969), 273-274.

23. W. J. Pervin, Quasi-uniformization of topological spaces, Math. Ann., 147 (1962), 316-317.

24. J. L. Sieber and W. J. Pervin, Completeness in quasi-uniform spaces, Math. Ann., 158 (1965), 79-81.

25. M. Sion and R. C. Willmott, Hausdorff measures on abstract spaces, Trans. Amer. Math. Soc., 123 (1966), 275-309.

26. C. Votaw, Uniqueness of compatible quasi-uniformities, Canad. Math. Bull., to appear.

Received August 11, 1971 and in revised form December 22, 1971.

Virginia Polytechnic Institute

AND STATE UNIVERSITY

Slippery Rock State College 



\section{PACIFIC JOURNAL OF MATHEMATICS}

\section{EDITORS}

\author{
H. SAMELSON \\ Stanford University \\ Stanford, California 94305 \\ C. R. HOBBY \\ University of Washington \\ Seattle, Washington 98105
}

\section{J. DugundjI}

Department of Mathematics University of Southern California Los Angeles, California 90007

\section{RICHARD ARENS}

University of California

Los Angeles, California 90024

\section{ASSOCIATE EDITORS}
E. F. BECKENBACH
B. H. NeumanN
F. WOLF
K. YosHIDA

\section{SUPPORTING INSTITUTIONS}

\author{
UNIVERSITY OF BRITISH COLUMBIA \\ CALIFORNIA INSTITUTE OF TECHNOLOGY \\ UNIVERSITY OF CALIFORNIA \\ MONTANA STATE UNIVERSITY \\ UNIVERSITY OF NEVADA \\ NEW MEXICO STATE UNIVERSITY \\ OREGON STATE UNIVERSITY \\ UNIVERSITY OF OREGON \\ OSAKA UNIVERSITY
}

\author{
UNIVERSITY OF SOUTHERN CALIFORNIA \\ STANFORD UNIVERSITY \\ UNIVERSITY OF TOKYO \\ UNIVERSITY OF UTAH \\ WASHINGTON STATE UNIVERSITY \\ UNIVERSITY OF WASHINGTON \\ ${ }^{*} \stackrel{*}{*}{ }^{*}$
AMERICAN MATHEMATICAL SOCIETY
NAVAL WEAPONS CENTER
}

The Supporting Institutions listed above contribute to the cost of publication of this Journal, but they are not owners or publishers and have no responsibility for its content or policies.

Mathematical papers intended for publication in the Pacific Journal of Mathematics should be in typed form or offset-reproduced, (not dittoed), double spaced with large margins. Underline Greek letters in red, German in green, and script in blue. The first paragraph or two must be capable of being used separately as a synopsis of the entire paper. The editorial "we" must not be used in the synopsis, and items of the bibliography should not be cited there unless absolutely necessary, in which case they must be identified by author and Journal, rather than by item number. Manuscripts, in duplicate if possible, may be sent to any one of the four editors. Please classify according to the scheme of Math. Rev. Index to Vol. 39. All other communications to the editors should be addressed to the managing editor, Richard Arens, University of California, Los Angeles, California, 90024.

50 reprints are provided free for each article; additional copies may be obtained at cost in multiples of 50 .

The Pacific Journal of Mathematics is published monthly. Effective with Volume 16 the price per volume (3 numbers) is $\$ 8.00$; single issues, $\$ 3.00$. Special price for current issues to individual faculty members of supporting institutions and to individual members of the American Mathematical Society: $\$ 4.00$ per volume; single issues $\$ 1.50$. Back numbers are available.

Subscriptions, orders for back numbers, and changes of address should be sent to Pacific Journal of Mathematics, 103 Highland Boulevard, Berke'ey, California, 94708.

PUBLISHED BY PACIFIC JOURNAL OF MATHEMATICS, A NON-PROFIT CORPORATION

Printed at Kokusai Bunken Insatsusha (International Academic Printing Co., Ltd.), 270, 3-chome Totsuka-cho, Shinjuku-ku, Tokyo 160, Japan. 


\section{Pacific Journal of Mathematics}

\section{Vol. 43, No. $3 \quad$ May, 1972}

Max K. Agoston, An obstruction to finding a fixed point free map on a manifold.... 543

Nadim A. Assad and William A. Kirk, Fixed point theorems for set-valued mappings

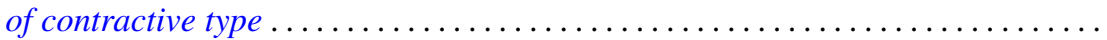

John Winston Bunce, Characterizations of amenable and strongly amenable

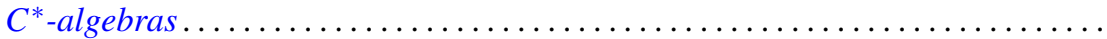

Erik Maurice Ellentuck and Alfred Berry Manaster, The decidability of a class of

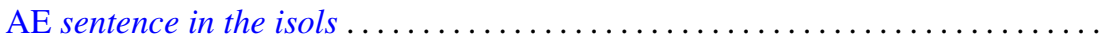

U. Haussmann, The inversion theorem and Plancherel's theorem in a Banach

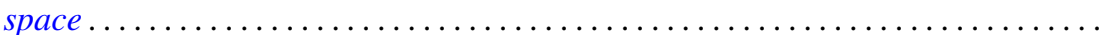

Peter Lawrence Falb and U. Haussmann, Bochner's theorem in infinite dimensions.

Peter Fletcher and William Lindgren, Quasi-uniformities with a transitive base ..... Dennis Garbanati and Robert Charles Thompson, Classes of unimodular abelian

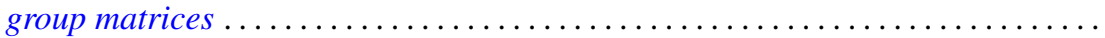

Kenneth Hardy and R. Grant Woods, On c-realcompact spaces and locally bounded normal functions

Manfred Knebusch, Alex I. Rosenberg and Roger P. Ware, Grothendieck and Witt rings of hermitian forms over Dedekind rings .......................

George M. Lewis, Cut loci of points at infinity.

Jerome Irving Malitz and William Nelson Reinhardt, A complete countable $L_{\omega_{1}}^{Q}$ theory with maximal models of many cardinalities . . . . . . . . . . . . . . . . .

Wilfred Dennis Pepe and William P. Ziemer, Slices, multiplicity, and Lebesgue

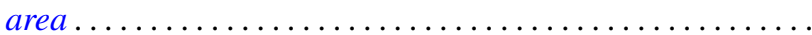

Keith Pierce, Amalgamating abelian ordered groups . .

Stephen James Pride, Residual properties of free groups . . . . . . . . . . . . . 725

Roy Martin Rakestraw, The convex cone of n-monotone functions .

T. Schwartzbauer, Entropy and approximation of measure preserving transformations .

Peter F. Stebe, Invariant functions of an iterative process for maximization of a polynomial...

Kondagunta Sundaresan and Wojbor Woyczynski, L-orthogonally scattered

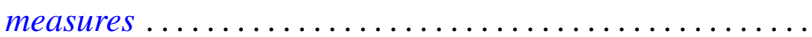

Kyle David Wallace, $C_{\lambda}$-groups and $\lambda$-basic subgroups $\ldots \ldots \ldots$

Barnet Mordecai Weinstock, Approximation by holomorphic functions on certain

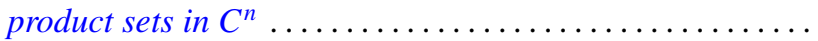

Donald Steven Passman, Corrections to: "Isomorphic groups and group rings”.

Don David Porter, Correction to: "Symplectic bordism, Stiefel-Whitney numbers, and a Novikov resolution"

John Ben Butler, Jr., Correction to: “Almost smooth perturbations of self-adjoint operators".

Constantine G. Lascarides, Correction to: "A study of certain sequence spaces of Maddox and a generalization of a theorem of Iyer" ...... ...

George A. Elliott, Correction to: "An extension of some results of takesaki in the reduction theory of von neumann algebras" ......................... 